\title{
Reasons for Discontinuation of Dantrolene in Persons with Brain Injuries with Spasticity: A Retrospective Review
}

\author{
Anne Kuwabara ${ }^{1 *}$, Benjamin Dirlikov ${ }^{2}$ and Kazuko Shem ${ }^{3}$ \\ ${ }^{1}$ Resident Physician, Stanford University, USA \\ ${ }^{2}$ Director of the Rehabilitation Research Center, Santa Clara Valley Medical Center, Rehabilitation Research Center, USA \\ ${ }^{3}$ Department of Physical Medicine and Rehabilitation, Santa Clara Valley Medical Center, USA
}

*Corresponding author: Anne Kuwabara MD, Resident Physician, Stanford University, Division of Physical Medicine and Rehabilitation, USA.

\begin{abstract}
Dantrolene is a muscle relaxant used to treat spasticity in persons with neurological injuries. At our acute rehabilitation unit, there have been anecdotal reports of patients developing dysphagia after starting dantrolene. The objective of this study is to investigate association between dantrolene and dysphagia in addition to other side effects of dantrolene. A retrospective chart review was performed on patients 18 years old or older that were started on dantrolene for spasticity during their acute rehabilitation admission. Exclusion criteria included any patients that have missing data, were started on dantrolene for reasons other than spasticity, or were started on dantrolene outside of their inpatient admission. Between 2015-2018, 62 out of 1,542 inpatients were started on dantrolene. Fifty patients tolerated the medication, and 12 patients discontinued the medication due to dysphagia ( $n=2)$, fatigue $(n=3)$, elevated LFTs $(n=1)$, lack of effect $(n=3)$, and other complications ( $n=3)$. Demographic comparisons and regression models revealed no significant predictors of dysphagia and other side effects. Relatively high percentage of patients (19.4\%) who were started on dantrolene discontinued the medication due to side effects or lack of efficacy. These findings can provide clinicians with insight into expected side effects when using dantrolene to treat spasticity.
\end{abstract}

Keywords: Brain; Depression; Muscle; Injuries; Spinal cord; Cerebral vascular; Heart failure; Neuroleptic

Abbreviations: CVA: Cerebral Vascular Accident; TBI: Traumatic Brain Injury; SCI: Spinal Cord Injury; SAH: Subarachnoid Hemorrhage; QD: Daily; BID: Twice a Day; TID: Three Times a Day; LFT: Liver Function Test; F: Female; M: Male

\section{Introduction}

Dantrolene is an oral medication that treats muscle spasticity in persons with neurological injuries, such as cerebrovascular accidents (CVA), traumatic brain injury (TBI), and spinal cord injury (SCI). Dantrolene reduces the amount of calcium released from the sarcoplasmic reticulum within muscle cells to decrease the force of muscle contraction [1,2]. It does not have any effect on the transmission of nerve impulses to the muscle [3]. It is also specific for skeletal muscle, with little demonstrated effect on cardiac and smooth muscle. Voluntary contractions are less inhibited than spastic tension or reflex contractions [3]. Its effect on voluntary muscle is substantial enough to require a 2-fold increase in voluntary effort to maintain similar muscle tension, but it does not produce complete muscle paralysis [3]. Dose-response studies in animals show a maximum of about $50 \%$ inhibition of muscle contraction [3]. Due to its direct action on muscle, dantrolene is thought to be less sedating. Therefore, it is often considered as a 
second-line spasticity medication to baclofen, which is the first-line drug for spasticity but is known to have significant sedating effects due to its mechanism of action in the central nervous system [4]. Side effects of dantrolene include drowsiness, dizziness, weakness, general malaise, fatigue, and diarrhea [5].

Since the 1970s there has been a paucity of research on dantrolene and spasticity, with few mentioning dysphagia as a possible side effect. Locatelli et al. published a case report of worsening dysphagia in a 12-year old child with a traumatic brain injury (TBI) [6]. After 5 days on dantrolene, the patient's dysphagia worsened and developed pharyngeal spasm. Dantrolene was suspected as causing the pharyngeal spasms and discontinued with subsequent improvement. At our acute rehabilitation unit, there have been anecdotal reports of patients with neurological injuries developing severe dysphagia (i.e. downgraded from a regular diet to nothing per os [NPO]) after starting dantrolene. The gap in knowledge of the association between dysphagia and dantrolene leaves providers without clear information to counsel patients. On one hand, dysphagia may increase the risk of aspiration and poor nutrition, but addressing and minimizing dysphagia may decrease associated complications (e.g. pneumonia), decrease hospital length of stay by expediting recovery through minimizing disruptions in nutrition, and improve patient's satisfaction [7]. On the other hand, ineffective spasticity treatment may decrease patient comfort and function, and put patients at higher risk of falls, pressure ulcers, and contractures [4]. Therefore, the goal of this study was to provide preliminary evidence in guiding spasticity treatment with dantrolene for persons with neurological injuries in the acute rehabilitation setting. We sought to explore whether there is an association between dantrolene and dysphagia and other side effects for not tolerating dantrolene.

\section{Methods}

This study was approved by the local Institutional Review
Board (IRB) in 2015. Between 2015-2018, a retrospective medical record review was performed on all patients with traumatic or non-traumatic brain injury who were admitted to our inpatient acute rehabilitation units and who were started on dantrolene for spasticity during their admission. Inclusion criteria included age 18-100 years old, diagnosis of spasticity related to neurologic injury, and started on dantrolene during their inpatient rehabilitation admission. Exclusion criteria included started dantrolene for reasons other than spasticity or started dantrolene prior to inpatient rehabilitation admission. The reason for discontinuation of dantrolene was recorded as the primary outcome measure. Written consent was not obtained because this was a chart review and deemed exempt per the Santa Clara Valley Medical Center IRB.

\section{Data analysis}

Demographic and medical characteristics were compared between individuals who continued and discontinued dantrolene treatment. Two binary logistic regressions were conducted to investigate demographic variables association with either 1) development of secondary complications or 2) development of dysphagia within the group with secondary complications.

\section{Results}

Medical records of 1,542 patients were reviewed, and 62 patients out of 1,542 were started on dantrolene during their inpatient rehabilitation admission (Table 1).

Fifty (80.6\%) tolerated the medication without any side effects. Twelve patients (19.4\%) discontinued dantrolene (Table 2) due to fatigue $(n=3)$, dysphagia $(n=2)$, lack of effect $(n=3)$, intermittent diplopia $(n=1)$, possible interaction with methotrexate $(n=1)$, and thrombocytopenia $(n=1)$, and elevated liver function test (LFT) $(\mathrm{n}=1)$. Demographic comparisons between individuals who did or did not continue taking dantrolene did not reveal any statistically significant differences (p>.28; Table 1) (Table 2).

Table 1: Demographics. Table 1 presents demographic characteristics for individuals who continued and discontinued dantrolene treatment. Age is presented in years and time from injury is presented in days, both include group mean and, in parentheses, standard deviation. All other variables represent counts.

\begin{tabular}{|c|c|c|c|c|}
\hline Variable & $\begin{array}{c}\text { Continued On Dantro- } \\
\text { lene } \mathrm{N}=50\end{array}$ & $\begin{array}{c}\text { Discontinued Dantro- } \\
\text { lene } \mathrm{N}=12\end{array}$ & Test Statistic & P-Value \\
\hline Male / Female & $30 / 20$ & $5 / 7$ & $\chi^{2}(1)=.011$ & 0.916 \\
\hline Ethnicity & & & White $v$ NW $\chi^{2}(1)=.799$ & 0.371 \\
\hline White & 18 & 6 & & \\
\hline Asian & 15 & 3 & & \\
\hline Hispanic & 6 & 1 & & \\
\hline African American / Black & 3 & 0 & & \\
\hline Other & 8 & 2 & & \\
\hline Etiology Non-Traumatic/Traumatic & $37 / 13$ & $5 / 7$ & $\chi^{2}(1)=1.153$ & 0.283 \\
\hline Mean age in years (STD) & $47.3(18.9)$ & $49.1(20.2)$ & $F(1,60)=.088$ & 0.768 \\
\hline Time from injury (days); mean (STD) & $65.4(47.4)$ & $49.1(42.3)$ & $F(1,60)=1.197$ & 0.278 \\
\hline
\end{tabular}


Table 2: Patients who stopped dantrolene due to side effects and lack of effect.

\begin{tabular}{|c|c|c|c|c|c|c|}
\hline Age & Sex & $\begin{array}{l}\text { Ethnic- } \\
\text { ity }\end{array}$ & Etiology & $\begin{array}{l}\text { Starting And Ending } \\
\text { Dose }\end{array}$ & Reason For Discontinuation & $\begin{array}{l}\text { Duration On Dantro- } \\
\text { lene }\end{array}$ \\
\hline 47 & $\mathrm{~F}$ & White & CVA & $25 \mathrm{mg}$ TID & Lack of effect & 1 day \\
\hline 55 & M & White & TBI & $\begin{array}{l}50 \mathrm{mg} \text { TID } \rightarrow 50 \mathrm{mg} \\
\text { BID }\end{array}$ & Lack of effect & 48 days \\
\hline 26 & $\mathrm{~F}$ & White & CVA \& SCI & $50 \mathrm{mg}$ QD $\rightarrow 75 \mathrm{mg}$ TID & Lack of effect & 7 days \\
\hline 62 & M & Asian & CVA & 25mg BID & Fatigue & 1 day \\
\hline 34 & M & Other & GBM & $25 \mathrm{mg}$ QD & Fatigue & 3 days \\
\hline 17 & M & White & TBI & $25 \mathrm{mg}$ TID & Fatigue & 2 days \\
\hline 57 & M & Hispanic & CVA & $25 \mathrm{mg}$ BID & Dysphagia & 2 days \\
\hline 80 & $\mathrm{~F}$ & Asian & CVA & $25 \mathrm{mg}$ QD & Dysphagia & 6 days \\
\hline 48 & $\mathrm{~F}$ & White & $\begin{array}{l}\text { TBI and SCI Bipolar } \\
\text { disorder }\end{array}$ & $25 \mathrm{mg}$ TID & Elevated LFTs & 20 days \\
\hline 72 & $\mathrm{~F}$ & Asian & SAH & $25 \mathrm{mg}$ BID & Intermittent diplopia & 16 days \\
\hline 62 & M & Other & CVA & $\begin{array}{l}25 \mathrm{mg} \text { BID } \rightarrow 25 \mathrm{mg} \\
\text { TID }\end{array}$ & $\begin{array}{l}\text { Noted interaction with metho- } \\
\text { trexate }\end{array}$ & 11 days \\
\hline 22 & M & White & TBI and SCI & $25 \mathrm{mg}$ TID & Thrombocytopenia & 4 days \\
\hline
\end{tabular}

Binary logistic regressions did not reveal any significant predictors (age, time from injury, time on dantrolene, sex, race, and etiology [traumatic vs. non-traumatic]) for discontinuing dantrolene treatment ( $p>$.27). Due to the small sample size, the logistic regression aimed to investigate risk factors associated with developing dysphagia (comparison with other complications; $\mathrm{N}=12$ ) failed due to limited cases per demographic variables.

Two patients (age 57 and 80) who discontinued dantrolene due to worsening of dysphagia both had CVA, and they discontinued dantrolene in 2 and 6 days. One patient was on mechanical soft diet, and the other was on dysphagia ground diet. Both were considered to have worsening dysphagia, yet the severity did not warrant a change in diet.

\section{Discussion}

The impetus of this study originated due to an anecdotal concern that dantrolene exacerbated dysphagia in patients with spasticity due to neurologic injuries at our acute inpatient rehabilitation center. From the Ryanodex prescribing information document, dysphagia was observed in $10 \%$ of healthy volunteers taking the medication[8]. In this study, only two patients (3.2\%) discontinued dantrolene due to dysphagia. Both patients had preexisting dysphagia but did not have severe enough worsening of dysphagia to change their diet level.

Besides dysphagia, other reasons for discontinuing dantrolene in this study included no effect on spasticity $(n=3)$, fatigue $(n=3)$, interaction with methotrexate $(n=1)$, elevated LFTs $(n=1)$, diplopia $(n=1)$, thrombocytopenia $(n=1)$. For the patients who discontinued dantrolene due to not achieving any effect for the treatment of spasticity, this could be because most patients never reached the maximum dose of $100 \mathrm{mg}$ four times per day or that they did not continue the medication for a long enough duration (i.e., one patient discontinued after one day). Recommended duration of treatment is at least seven days to assess full effect according to Micromedex ®. It is unclear if the dantrolene could have had more effective if these patients had extended their dantrolene treatment.

Three patients $(4.8 \%)$ had dantrolene discontinued due to fatigue, which is much lower rate compared to the adverse event of "somnolence" noted in $17 \%$ of patients in IBM Micromedex $®[9]$. This finding was confounded as all patients were concurrently on baclofen, which is well known to cause sedation. It has been reported that dantrolene can increased central nervous system (CNS) depression while patients are on other muscle relaxants and pain medications [2]. If these classes of medications are being prescribed concurrently, somnolence should be monitored more closely when starting dantrolene.

One patient also had their dantrolene discontinued due to potential drug reactions with methotrexate (MTX). The interaction results in an increased risk of methotrexate toxicity and hepatotoxicity [2]. Dantrolene also interacts with several medications resulting in severe consequences. For example, concomitant hepatotoxic drugs can result in liver failure, calcium channel blockers (e.g. amlodipine) can result in severe hyperkalemia with cardiovascular collapse, and central acting muscle relaxants (carisoprodol, barbiturates, benzodiazepines) can result in central nervous system and respiratory depression.

Dantrolene is also known to cause liver injury and increase in the levels of AST and ALT [2]. Mild, asymptomatic serum aminotransferase elevations during dantrolene therapy are relatively uncommon (1\%), but clinically overt liver injury is estimated to occur in 1 to 2 per thousand treated persons $(0.1 \%$ to $0.2 \%$ [ [10]. The two patients (3.2\%) who had dantrolene discontinued due to elevated LFTs also concurrently had pancreatitis or were on medications metabolized by the liver for bipolar disorder. Dantrolene may need to be avoided in patients 
with active liver pathologies and/or on hepatotoxic medications as described above.

There was one patient who experienced thrombocytopenia that was attributed to dantrolene or fluoxetine as the patient started both medications in a similar time frame. Although very rare, thrombocytopenia is a reported side effect of dantrolene [2]. Heparin-induced thrombocytopenia and serotonin release assay antibodies were negative in these patients. Complete blood count and basic metabolic panel may need to be checked along with LFTs when first starting dantrolene, changing the dose, or starting a new medication [11].

Other adverse effects of dantrolene that were not seen in our population are flushing (27\%), diarrhea, nausea $(10 \%)$ and more serious side effects such as heart failure, tachycardia (3\%), gastrointestinal hemorrhage, aplastic anemia, leukopenia, small lymphocytic lymphoma, anaphylaxis, respiratory insufficiency [2]. Dantrolene is also used to treat neuroleptic malignant syndrome, in which case dantrolene is administered parenterally, and the most common side effects in the form of administration were muscle weakness in $22 \%$, phlebitis in $10 \%$, respiratory failure in $3 \%$, and gastrointestinal discomfort in $3 \%$, which were not observed in this study [11].

\section{Conclusions}

The findings from this study help characterize the reasons for discontinuing dantrolene and provide clinicians with further insight into which patients may be appropriate candidates for dantrolene. Due to dantrolene's effect on skeletal muscle, dantrolene may exacerbate preexisting dysphagia, yet more work directly investigating this theory is required. Patients who experience fatigue with dantrolene should be evaluated for other possible causes such as concurrent use of other sedating medications. Patients should have their liver function tests checked when starting dantrolene and when changing dose. Future prospective studies with larger sample sizes are needed to understand risk factors for secondary complications related to dantrolene.

\section{Limitations}

This study was limited by its small sample size, which in part was due to its single site retrospective study design. The retrospective nature of the study is also a limitation due to possible selection bias and inability to determine causation. Additionally, including individuals who developed dysphagia but were not on dantrolene may help understand the risk of developing dysphagia when on dantrolene.

\section{Acknowledgement}

None.

\section{Conflict of Interest}

No conflict of interest.

\section{References}

1. Kheder A, Krishnan Padmakumari Sivaraman Nair (2012) Spasticity: pathophysiology, evaluation, and management. Pract Neurol 12: 289298.

2. Dantrolene Sodium (2017) In: In Depth Answers [database on the Internet]. Greenwood Village (CO): Truven Health Analytics. Available from: www.micromedexsolutions.com

3. Pinder RM, Brogden RN, Speigh TM, G S Avery (1977) Dantrolene sodium: a review of its pharmacological properties and therapeutic efficacy in spasticity. Drugs 13(1): 3-23.

4. Chang E, Ghosh N, Yanni D, Sujin Lee, Daniela Alexandru, et al. (2013) A Review of Spasticity Treatments: Pharmacological and Interventional Approaches. Crit Rev Phys Rehabil Med 25(1-2): 11-22.

5. FDA/CEDR resources page (2020) Food and Drug Administration Web site. https://www.accessdata.fda.gov/drugsatfda_docs/label/2012/017443s043s046s048s049lbl.pdf.

6. Locatelli F, Pozzi M, Avantaggiato P, Lanfranchi M, Tufarulo L, et al. (2014) Pharyngeal spasticity due to dantrolene. J Clin Pharm Ther 39(4): 449-551.

7. Schwarz M, Coccetti A, Murdoch A, Elizabeth Cardell (2018) The impact of aspiration pneumonia and nasogastric feeding on clinical outcomes in stroke patients: A retrospective cohort study. J Clin Nurs 27(1-2): e235-e241.

8. (2014) Product Information: RYANODEX(R) intravenous injection suspension, dantrolene sodium intravenous injection suspension. Eagle Pharmaceuticals, Inc. (per manufacturer), Woodcliff Lake, NJ

9. Dantrolene (2020) In: LiverTox: Clinical and Research Information on Drug-Induced Liver Injury. National Institute of Diabetes and Digestive and Kidney Diseases; 2012.

10. FDA/CEDR resources page (2012) Food and Drug Administration Web site. https://www.accessdata.fda.gov/drugsatfda_docs/label/2012/017443s043s046s048s049lbl.pdf.

11. Krause T, Gerbershagen MU, Fiege M, R Weisshorn, F Wappler (2004) Dantrolene - A review of its pharmacology, therapeutic use and new developments. Anaesthesia 59(4): 364-373. 